Historic, archived document

Do not assume content reflects current scientific knowledge, policies, or practices. 



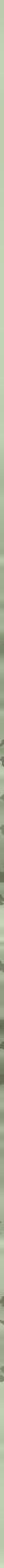


This publication is the result of the efforts of many people distributed over 23 years. The plants named herein represent 58 different families, all present in some degree on the 4600-acre San Joaquin Experimental Range. Range research on any area involves many things, but high in priority always is the discovery, identification, and evaluation of plant specles. This is frequently a continuing chore because conditions favorable to all plants seldom if ever occur, and many plants are so shortlived they are difficult to ind. Thus the long perlod required to develop this list.

It Is my hope, and I know the authors concur, that this publication will be of use to technicians and administrators.

E. Jo Woolfolk, Chief

Division of Range Management Research

Agriculture-Forest Service, Berkeley, Californla 


\section{A CHECK LIST OF PLANTS OF THE \\ SAN JOAQUIN EXPERIMENTAL RANGE}

by

Robert F. Buttery, Range Conservationist,
Division of Range Management Research,
and Lisle R. Greenl

Miscellaneous Paper No. 23

CALIFORNIA FOREST AND RANGE EXPERIMENT STATION

BERKELEY, CALIFORNIA

FOREST SERVICE--U.S. DEPARTMENT OF AGRICULTURE

If Formerly range conservationist, California Forest and Range Experiment Station; present address: California State Polytechnic College, San Luis Obispo, California. 



\section{PREFACE}

The San Joaquin Experimental Range is located near the center of the State in the granite-soil section of the Sierra Nevada foothills. It is in Madera County, 28 miles north of Fresno, California. This 4600-acre experimental area was established in 1934 as a branch station of the California Forest and Range Experiment Station, U. S. Forest Service. Research aimed at increased production from California foothill range through improved management, fertilization, brush control, and livestock management has been carried out since establishment.

The area lies in the "annual grass type" and is characterized by grassy rolling hills with a sparse covering of trees and occasional dense stands of brush. It is in the lower part of the woodland zone between the treeless valley floor and the higher brush and timber belts.

The herbaceous ground cover consists mostly of annual grasses and forbs. In contrast, perennials dominate most western range areas. Ecologically speaking, the area is near the lower limits of the Upper Sonoran Zone. The elevation varies from 700 to 1700 feet, with most of the area lying between 1000 and 1500 feet. Exposures are generally southwesterly.

The drainage basin empties into a small tributary of the San Joaquin River. No permanent streams flow through the area, but springs are numerous and the small drainages carry surface flows during the winter months.

The slope soils are shallow, residual, of granitic origin, and are classed in the Vista series. The swale soils are deeper, alluvial, and are classed in the Visalia series. Both have a low water-holding capacity.

The climate is characterized by mild, rainy winters and hot, dry summers. Snow is rare. Seasonal precipitation usually begins in September and has averaged 19.4 inches, with extremes of 12.3 and 32.1 inches, during the past 23 years. On the average, over 70 percent of the precipitation falls during 4 months, December through March. From mid-May to mid-September there is practically no rain at all.

Seeds of most herbaceous plant species germinate with the first $1 / 2$ to 1 inch of fall rain and grow slowly during the winter. The plants grow rapidly when warm temperatures return in March, reach maturity in April, and are mostly dry by mid-May. 
Plant species listed in the text have been collected at the Range during the past 23 years. Standards of abundance for herbaceous vegetation are as follows:

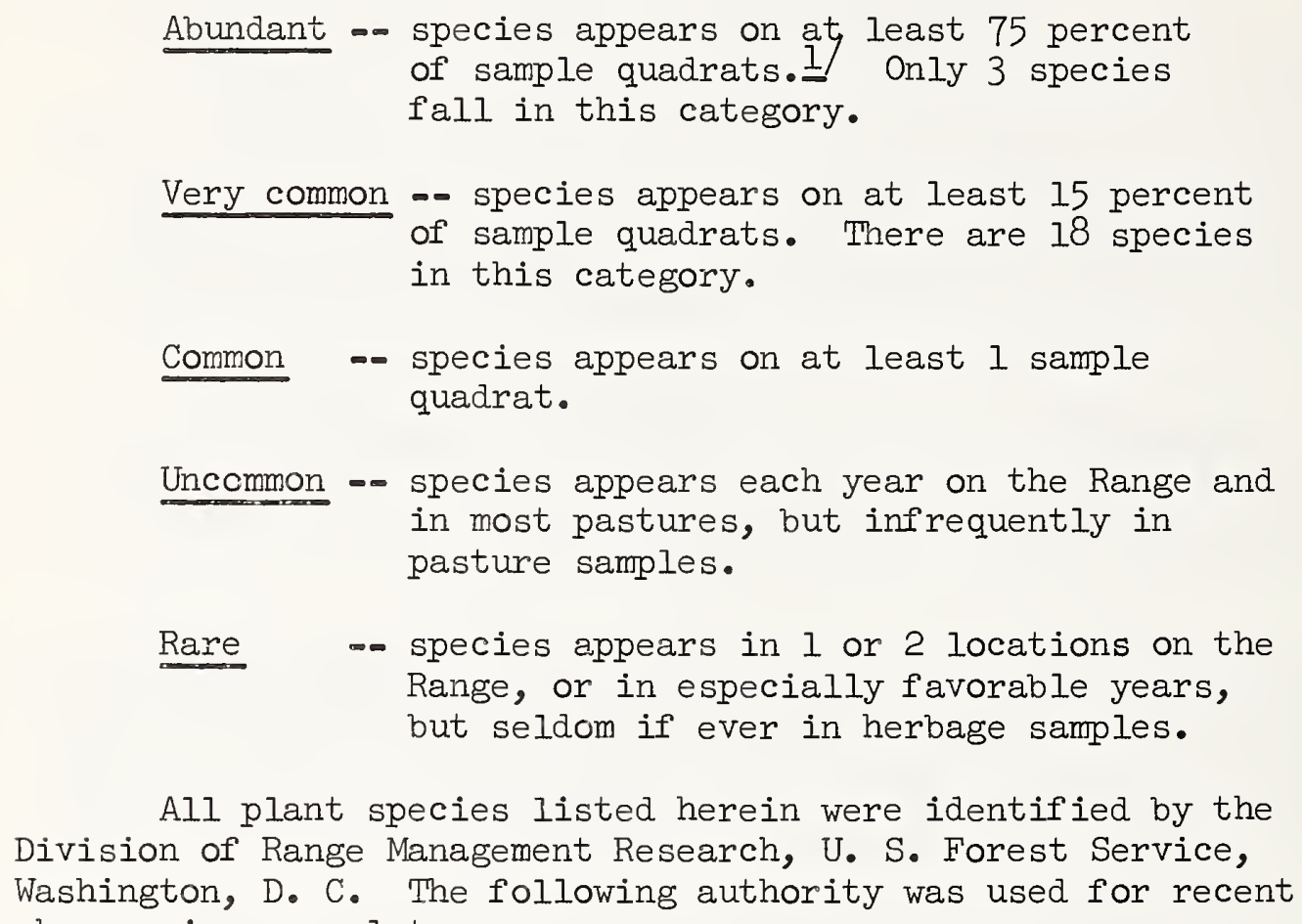

Rare - species appears in 1 or 2 locations on the Range, or in especially favorable years, but seldom if ever in herbage samples.

All plant species listed herein were identified by the Division of Range Management Research, U. S. Forest Service, Washington, D. C. The following authority was used for recent changes in nomenclature:

Abrams, Leroy. 1951. Illustrated Flora of the Pacific States. Stanford University Press, Stanford, California.

Botanical names in parentheses are synonyms which have been used at the Range. The following source was used for common names:

Kelsey, H. P., and Dayton, W. A. 1942. Standardized

Plant Names. Second Edition. J. Horace McFarland Co., Harrisburg, Pennsylvania. $675 \mathrm{p}$.

Locally well established common names are also listed for some plants. 
Grasses and grass-like plants .......................... Cyperaceae ................................... Sedge

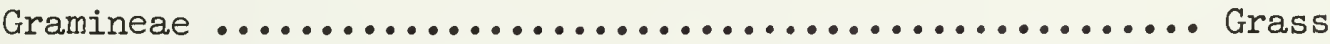

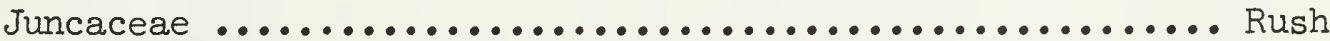

Lemnaceae ............................. Duckweed Typhaceae $\ldots \ldots \ldots \ldots \ldots \ldots \ldots \ldots \ldots \ldots \ldots \ldots \ldots$ Cattail

Forbs $\ldots \ldots \ldots \ldots \ldots \ldots \ldots \ldots \ldots \ldots \ldots \ldots \ldots \ldots \ldots \ldots \ldots \ldots \ldots \ldots \ldots \ldots \ldots \ldots \ldots \ldots \ldots \ldots \ldots$

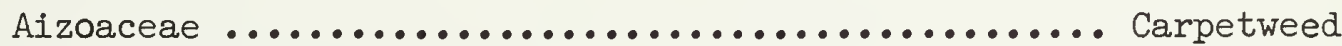

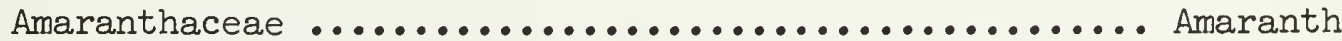

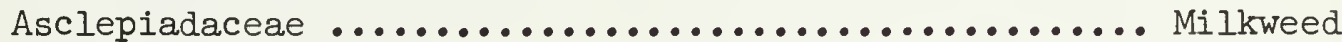

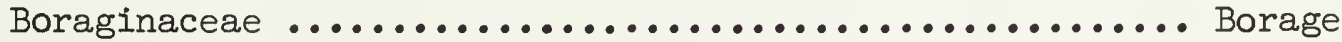

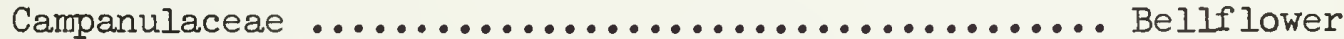

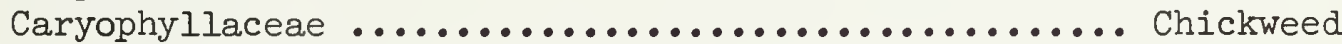

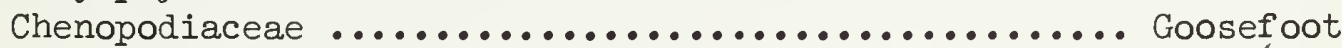

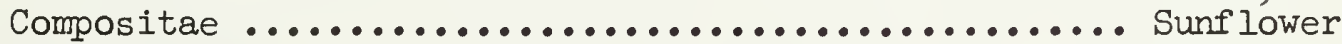

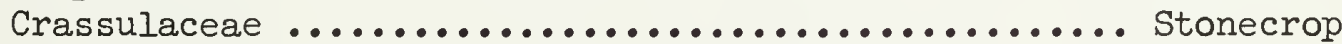

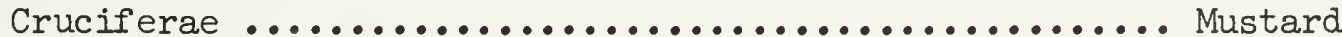

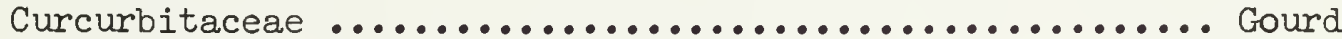

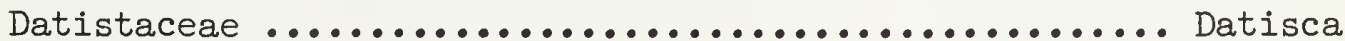

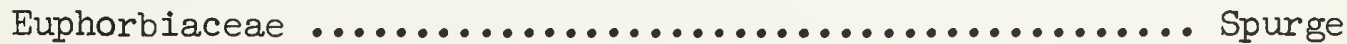

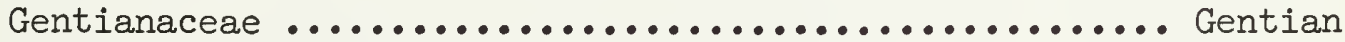

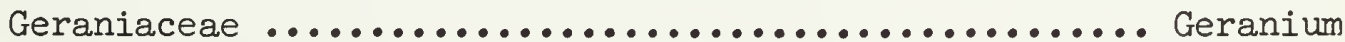

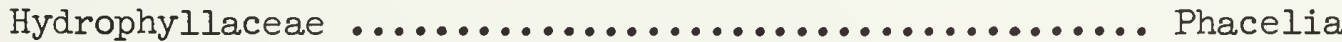
Labiatae .............................. Mint Leguminosae ................................. Pea Liliaceae .................................. Lily

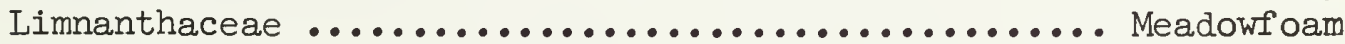
Linaceae .................................. Flax

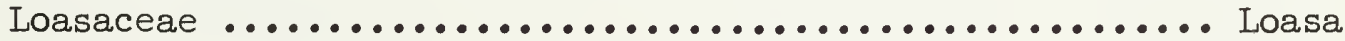

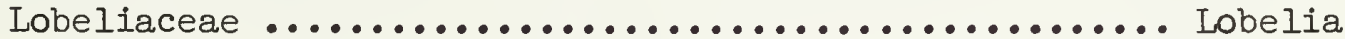

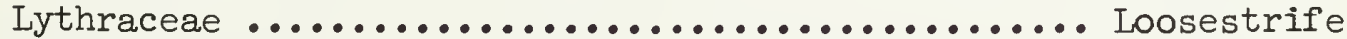

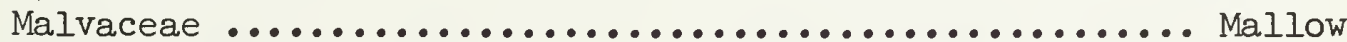

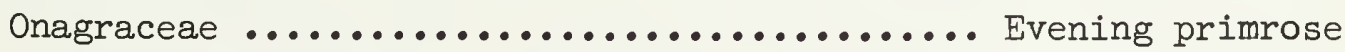

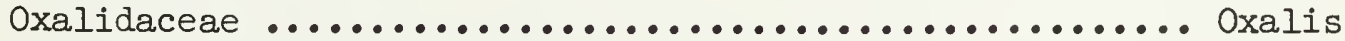

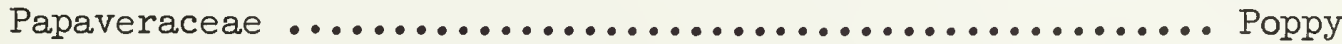

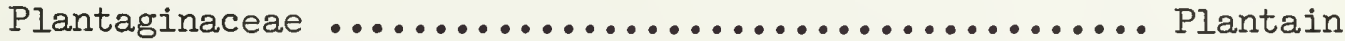

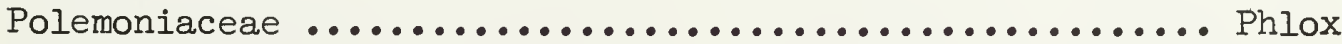

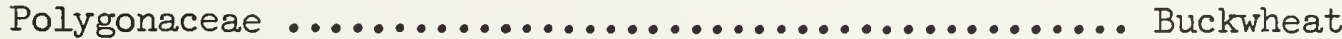

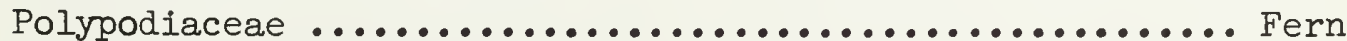
Portulacaceae ............................... Purslane Primulaceae ............................... Primrose

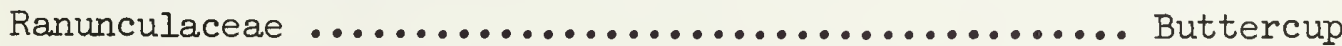
Rosaceae ................................. Rose

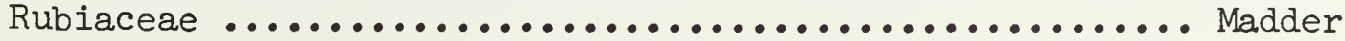




\section{Forbs (Continued)}

Saxifragaceae ............................ Saxifrage

Scrophulariaceae ........................... Figwort

Solanaceae .............................. Nightshade

Umbelliferae ............................... Parsley

Valerianaceae ............................... Valerian

Verbenaceae ................................... Verbena

Woody plants ............................................. Anacardiaceae ............................... Sumac Caprifoliaceae ........................... Honeysuckle 30

Ericaceae ................................... Heath 30 32

Fagaceae ...................................... Oak

Hydrophyllaceae ............................ Phacelia

Loranthaceae .............................. Mistletoe

Pinaceae ..................................... Pine

Rhamnaceae ............................. Buckthorn

Rubiaceae ............................... Madder

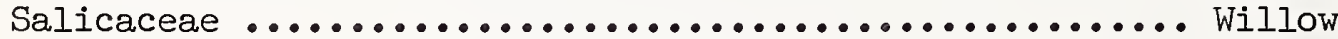
31

30 
GRASSES AND GRASS-LIKE PLANTS

Botanical and common name

$\begin{array}{ll}: & \text { Occurrence } \\ : & :\end{array}$

\section{TYPHACEAE Cattail family}

Typha latifolia L. Common cattail

Rare

Wet swale

Broad-leaved cattail

GRAMINEAE Grass family

Aira caryophyllea L.

(Aspris caryophyllea Nash)

Silver hairgrass

Avena barbata Brot.

Slender wild oat

Common

Avena fatua L.

Wild oat

Briza minor L.

Little quakinggrass

Common

Swale

Bromus arenarius Labill.

Australian brome

Common

Bromus mollis L.

(Bromus hordeaceus L.)

Sof t brome or chess

Bromus rigidus Roth. Ripgut brome

Very common

Bromus rubens I.

Foxtail brome

Red brome

Abundant

Cynodon dactylon (I.) Pers.

Bermuda grass

Very common

Rare

Slope, on shallow soil

Slope, especially south

Slope

Slope, especially south

Slope and dry swale

Slope, especially shaded, and dry swale

Very common

Slope, all exposures

Uncommon

Wet swale

Deschampsia danthonioides

(Trin.) Munro ex Benth.

Annual hairgrass

Uncommon

Lower slope and swale

Digitaria sanguinalis (L.) Scop. Syntherisma sanguinalis Dulae,

Uncommon

Gardens

Fl. Haut.

Hairy crabgrass 
GRASSES AND GRASS-LIKE PLANTS

Botanical and common name $\begin{array}{llll}: & \text { Occurrence }: & : & \text { Habitat } \\ & : & & \end{array}$

$\begin{array}{lll}\text { Distichlis stricta (Torr॰) Rydb. } & \text { Rare } & \text { Creek bank }\end{array}$

Elymus triticoides Buckl.

Creeping wildrye

Beardless wildrye

Eragrostis cilianensis (All.)

Iutati

Rare

Dry swale

Stinkgrass

Eragrostis diffusa Buckl。 Spreading lovegrass

Festuca confusa Piper

Klickitat fescue

Hairy-leaved fescue

Festuca grayi (Abrams) Piper Gray's fescue

Festuca megalura Nutt。 Foxtail fescue

Festuca pacifica Piper Pacific fescue

Festuca reflexa Buckl. Twoflower fescue

Gastridium ventricosum (Gouan)

Schinz and Thell

Nitgrass

Hordeum brachyantherum Nevski (Hordeum nodosum I.) Meadow barley

Hordeum hystrix Roth. (Hordeum gussonianum Parl.) Mediterranean barley

Rare

Barnyard

Uncommon

Slope

Common

Abundant

Unc ommon

Unc ommon

Unc ommon

Barnyard and garden

Slope, especially south

Slope, all exposures and dry swale

Slope, especially north

Slope, especially south

Common

Slope, on shallow soil

Dry swale

Very common Wet swale 


\begin{tabular}{llll}
\hline Botanical and common name & $:$ & Occurrence & $:$ \\
& $:$ & & \\
\hline
\end{tabular}

Hordeum leporinum Link

(Hordeum murinum L.)

Mouse barley

Common foxtail

Koeleria phleoides (Vill.) Pers. Annual koeleria

Lolium multiflorum Lam. Italian ryegrass

Annual ryegrass

Melica californica Scribn. California melicgrass

Melica imperfecta Trin.

Coastrange melicgrass

Small-flowered melicgrass

Oryzopsis miliacea (I。) Benth. Smilo

Panicum capillare occidentale Rydb.

Common witchgrass

Phalaris californica Hook. and Arn. California canarygrass

Phalaris caroliniana Walt. Carolina canarygrass

Phalaris coerulescens Sunol grass

Phalaris tuberosa stenoptera (Hack.) Hitchc. Hardinggrass

Poa annua $\mathrm{I}$. Annual bluegrass
Seeded

Common

Rare

Seeded

Uncommon

Uncommon

Seeded

Uncommon

Disturbed soil or lower slope

Swale

Swale and lower slope

Rock outcrop

Rock outcrop

Rare

Uncommon

Wet swale

Lower slope and swale

Seeded

Swale

Common

Swale 


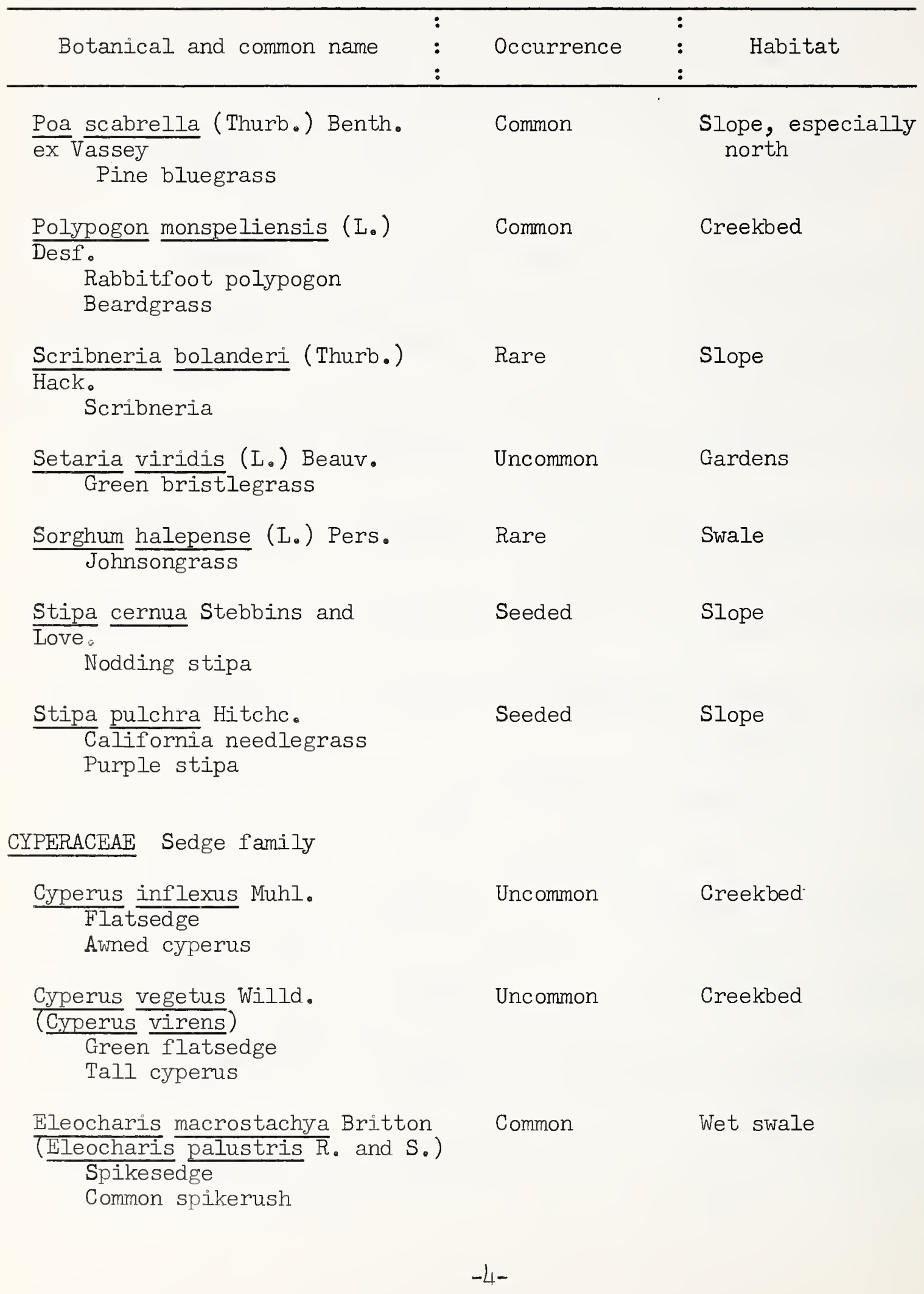


GRASSES AND GRASS-IIKE PLANTS

Botanical and common name

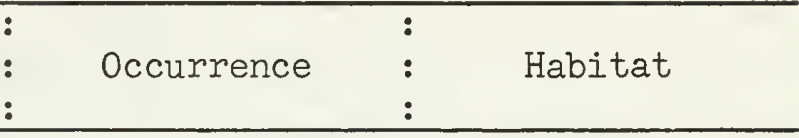

LEMNACEAE Duckweed family

Lemna sp.

Duckweed

Uncommon Seep

JUNCACEAE Rush family

Juncus bufonius L.

Common

Dry swale

Toad rush

Juncus oxymeris Engelm.

Slenderleaf rush

Common Wet swale

Pointed rush

Juncus macrophyllus Coville

Iong-leaved rush

Common

Wet swale 


\section{FORBS}

\begin{tabular}{llll}
\hline Botanical and common name & $:$ & $:$ & \\
& $:$ & & Occurrence \\
\hline
\end{tabular}

POLYPODIACEAE Fern family

Pityrogramma triangularis

(Kaulf.) Maxon

(Gymnogramme triangularis Kaulf.) outcrop Goldfern

Pellaea mucronata (D.C. Eaton)

D.C.Eaton

Birdsfoot cliffbrake

Common

Shaded rock outcrop

\section{LILIACEAE Lily family}

Allium hyalinum Curran

Uncommon

Slope

Eldorado onion

Paper-flowered onion

Brodiaea capitata Benth。

Dichelostemma capitatum

Very common Slope

[Benth.] Wood)

Bluedicks brodiea

Common brodiea

Brodiaea coronaria (Salisb.)

Common

Engler

Harvest brodiea

Brodiaea laxa (Benth.) Wats.

(Triteleia laxa Benth.)

Uncommon

Slope

Grassnut brodiea

Brodiaea volubilis (Kell.) Baker

(Dichelostemma californicum

[Torr.] Wood)

Vine brodiea

Twining brodiea

Snake lily

$\frac{\text { Calochortus }}{\text { Yellow mariposa }}$ Dougl.

Uncommon

Swale and

lower slope

Uncommon

Steep rocky slope 


\section{FORBS}

\begin{tabular}{llll}
\hline Botanical and common name & $\vdots$ & Occurrence & $\vdots$ \\
& $:$ & & Habitat \\
\hline
\end{tabular}

Calochortus luteus oculatus Wats. Uncommon Slope Mariposa

Chlorogalum pomeridianum (DC.) Common Rock outcrop Kunth.

Common soapplant

Amole soapplant

Muilla tenuis Congdon

(Muilla maritima [Torr.] Wats.) Nuilla

Common muilla

POLYGONACEAE Buckwheat family

Chorizanthe membranacea Benth.

Pink chorizanthe

Common

Slope, on

shallow soil

Spineflower

Chorizanthe stellulata Benth.

Starlet chorizanthe

Common

Slope

Spineflower

Common

Slope, on

shallow soil

Eriogonum gracillimum Wats.

Slender eriogonum

Eriogonum nudum Benth.

Barestem eriogonum

Eriogonum virgatum Benth. Virgate eriogonum

Polygonum aviculare L. Prostrate knotweed Knotweed

Uncommon

Slope

Knotgrass

Uncommon

Slope

Common

Slope

Uncommon

Barnyard and gardens

Polygonum punctatum Elliot

(Polygonum acre $\mathrm{H}_{\bullet} \mathrm{B}, \mathrm{K}_{\bullet}$ )

Uncommon

Creekbed

Dotted smartweed

Water smartweed 
FORBS

Botanical and common name

Pterostegia drymarioides

Fisch. and Mey.

Pterostegia

Rumex crispus L。 Curly dock

CHENOPODEACEAE Goosefoot family

Chenopodium album L. Lambsquarters goosefoot

Chenopodium ambrosiordes chilense (Schrad॰) Spegaz。 Wormseed goosefoot Pigweed

Chenopodium leptophyllum Nutt. Slimleaf goosefoot

Chenopodium pumilio $\mathrm{R}, \mathrm{Br}$. Goosefoot

\section{AMARANTHACEAE Amaranth family}

Amaranthus graecizans L。 Tumbleweed amaranthus

Tumbling pigweed

Amaranthus retroflexus L. Redroot amaranthus

Rough pigweed

\section{AIZOACEAE Carpetweed family}

Mollugo verticillata L。 Carpetweed

Indian chickweed
Common

Shaded slope

Common

Creekbed

Uncommon

Disturbed soil

Uncommon

Disturbed soil

Common

Disturbed soil

Uncommon

Disturbed soil

Uncommon

Disturbed soil

Uncommon

Disturbed soil 
FORBS

Botanical and common name $\quad: \begin{array}{ll}: \\ :\end{array}$

PORTULACACEAE Purslane family

Calindrina caulescens

menziesii Gray

(C. ciliata var. menziesii

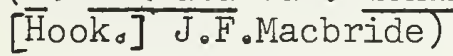
Redmaids

Montia fontana I. Indian lettuce

Water chickweed

Common

Wet swale

Montia perfoliata (Donn.) Howell Common Shaded slope Indian lettuce Miners' lettuce

Portulaca oleracea L. Common purslane

Very common Slope

CARYOPHYLILACEAE Chickweed family

Arenaria douglasii Fenzl. Douglas sandwort

Cerastium viscosum I.

Sticky cerastium

Mouse-ear chickweed

Herniaria cinerea DC.

Gray burstwort

Gray herniaria

Uncommon Gardens

Sagina apetala barbata Fenzl. Dwarf pearlwort

Unc ommon Slope

Common

Lower slope

Silene gallica L。

French silene

Common catchfly

Windmill pink

Uncommon Slope

$\frac{\text { Spergula arvensis L。 }}{\text { Corn }} \frac{\text { arpurry }}{\text { spura }}$

Unc ommon

Lower slope and

dry swale 
FORBS

\begin{tabular}{llll}
\hline Botanical and common name & $:$ & Occurrence & $\vdots$ \\
& $:$ & & Habitat \\
\hline
\end{tabular}

Spergularia marina (L.) Griseb.

(S. salina $\mathrm{J}_{\text {. and }} \mathrm{C}_{0}$ )

Saltmarsh sand spurry

Stellaria media (I.) Cyrill. Chickweed

Uncommon

Lower slope

Common

Lower slope

RANUNCULACEAE Buttercup family

Delphinium decórum patens

(Benth.) Grey.

Uncommon

Slope, especially north

Yellowtinge larkspur

Coast larkspur

Delphinium hanseni Greene

Hansen larkspur

Common

Shaded lower

slope

Ranunculus aquatilis L.

Common

Creekbed

(R. aquatilis var. capillaceus

[Thuill.] DC.)

Watercrowfoot buttercup

Water buttercup

Ranunculus californicus Benth.

California buttercup

Ranunculus hebecarpus Hook.

and Arn.

Pubescent-fruited buttercup

Common

Wet swale

Uncommon

Shady slope

PAPAVERACEAE Poppy family

Eschscholtzia caespitosa Benth. Uncommon Gold poppy

Common

Eschscholzia californica Cham.

Califormia poppy

Eschscholzia lobbii Greene

Fryingpans

Common

Slope, especially south

Slope, especially south

Slope 


\section{FORBS}

\begin{tabular}{llll}
\hline Botanical and common name & $:$ & $:$ & \\
& $:$ & & Occurrence \\
\hline
\end{tabular}

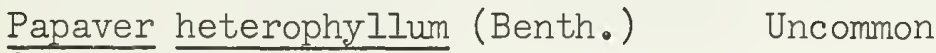

Shady north slope Greene Wind poppy

Platystemon californicus Benth. Creamcups platystemon

Common

Creekbank

CRUCIFERAE Mustard family

Arabis maxima Greene

Uncommon Rocky slope

Arabis arcuata Gray

(Arabis holboellii var.

arcuata Jepson)

Rockcress

Athysanus pusillus (Hook.

Greene

Dwarf athysanus

Capsella bursa-pastoris ( $\mathrm{L}_{0}$ )

Medic.

Shepherdspurse

Lepidium nitidum Nutt.

Tongue pepperweed

Common

Swale

Shining peppergrass

Radicula nasturtium-

aquaticum (L.) Britt, and

Rendle

Watercress

Sinapsis arvensis L.

$\frac{\text { (Brassica }}{\text { Field }} \frac{\text { arvensis }}{\text { charlock }}[$ L.] B.S.P.)

Common Slope

Common

Slope

Streptanthus coulteri (Wats.)

Common

Seep

Greene

Twistflower

Streptanthus diversifolius

Wats。

Uncommon

Seep

Uncommon

Creekbank

Varied-leaved streptanthus

Uncommon

Slope 


\section{FORBS}

\begin{tabular}{llll}
\hline Botanical and common name & $:$ & Occurrence & $\vdots$ \\
& $:$ & & \\
\hline
\end{tabular}

Streptanthus tortuosus Kell. Mountain streptanthus

Uncommon

Slope

Streptanthus heterophyllus Nutt. Uncommon

Slope San Diego streptanthus

Common

Slope Hairy fringepod

Tropidocarpum gracile Hook. Dobie pod

Common

Slope

\section{CRASSULACEAE Stonecrop family}

Tillaea erecta Hook. and Arn. Sand pygmyweed

Very common Slope

\section{SAXIFRAGACEAE Saxifrage family}

Lithophragma heterophylla

(Hooke and Arn。) Torr. and Gray. Hillstar

Common

Shady north slope

Saxifraga californica Greene

California saxifrage

Uncommon

Slope, especially north

Saxifraga integrifolia Hook. Hooker's saxifrage

Uncommon

Slope, especially north

ROSACEAE Rose family

Alchemilla occidentalis Nutt.

(A. arvensis [L。] Scop.)

Common

Slope

Field ladysmantle

Western ladysmantle 


\section{FORBS}

Botanical and common name

$\begin{array}{ll}: & \\ : & \text { Occurrence }\end{array}$

LEGUMINOSAE Pea family

Astragalus asymmetricus Sheldon

Rare

Slope
A. leucophyllus Torr. and Gray) Woolyleaf loco San Joaquin locoweed

Astragalus gambellianus Sheldon Common

(A. nigrescens Nutt.) Gambel's dwarf locoweed

Habitat

Lotus americanus (Nutt.) Bisch.
(Hosackia americana [Nutt.] Piper)

Very common

Lower slope

Spanish clover

Deervetch

Lotus argophylius decorus

(Jtn.) 0ttley

Uncommon

Slope

(Hosackia argophylla Gray)

Silver deervetch

Lotus micranthus Benth.

(Hosackia parviflora Benth.)

Uncommon Slope

Littleflower deervetch

Lotus scoparius (Nutt.) Ottley

(Hosackia glabra [Vogel] Torr.)

Uncommon

Slope, especially north

Broom deervetch

Deerweed

Lotus strigosus (Nutt.) Greene

(Hosackia strigosa Nutt.)

Common

Fine-leaved deervetch

Lotus subpinnatus Lag.

(Hosackia subpinnata [Lag.]

Torr. and Gray)

Chilean deervetch

Hairy lotus

Lupinus albifrons Benth.

Whiteface lupine

Common

Rocky slope

Bush lupine

Common

Slope

Slope, especially south 
FORBS

Botanical and common name

\begin{tabular}{lll}
$:$ & Occurrence & $:$ \\
$:$ & Habitat \\
\hline
\end{tabular}

Iupinus benthami Hel.

Common

Slope

Spider lupine

Bentham's lupine

Lupinus bicolor Lindl.

Bicolor lupine

Ground lupine

Iupinus densiflorus Benth.

Gully lupine

Uncommon

Slope

Dense-flowered lupine

Lupinus formosus Greene

Iunara lupine

Very common Slope

Summer lupine

Lupinus nanus Dougl.

Sky Iupine

Common

Slope

Medicago apiculata Willd.

Smoothbur medic

Uncommon

Lower slope and dry swale

Medicago hispida Gaertn.

California burclover

Uncommon, except: as seeded

Slope

Trifolium albopurpureum

Uncommon

Slope

Torr. and Gray.

Rancheria clover

Indian clover

Trifolium amplectens

Torr. and Gray.

Uncommon

Slope

Bladder clover

$\mathrm{Pale}$ sack clover

Trifolium ciliolatum Benth.

(Trifolium ciliatum Nutto)

Common

Lower slope

Foothill clover

Tree clover

Trifolium depauperatum

Depauperate clover

Uncommon

Slope

Dwarf sack clover 
FORBS

\begin{tabular}{|c|c|c|c|}
\hline Botanical and common name & $\begin{array}{l}: \\
: \\
:\end{array}$ & Occurrence & Habitat \\
\hline $\begin{array}{l}\frac{\text { Trifolium dichotomum Hook. }}{\text { and Arn. }} \\
\text { Branched Indian clover }\end{array}$ & & Uncommon & $\begin{array}{l}\text { Slope, especially } \\
\text { north }\end{array}$ \\
\hline$\frac{\text { Trifolium hirtum }}{\text { Rose }}$ clover & & Where seeded & Lower slope \\
\hline$\frac{\text { Trifolium incarnatum }}{\text { Crimson clover }}$ & & Where seeded & Dry swale \\
\hline$\frac{\text { Trifolium microcephalum }}{\text { Littlehead clover }}$ Pursh. & & Very common & Slope \\
\hline $\begin{array}{l}\frac{\text { Trifolium }}{\text { Clammy }} \text { obtusiflorum Hook. } \\
\text { Creek clover }\end{array}$ & & Uncommon & Creekbed \\
\hline$\frac{\text { Trifolium }}{\text { Subterranean clover }}$ & & Where seeded & Dry swale \\
\hline$\frac{\text { Trifolium }}{\text { Tomcat }} \frac{\text { tridentatum Lind. }}{\text { clover }}$ & & Common & Lower slope \\
\hline$\frac{\text { Trifolium }}{\text { Whitetip clover }}$ variegatum & & Very common & Wet swale \\
\hline LINACEAE Flax family & & & \\
\hline$\frac{\text { Linum }}{\text { Common flax }} \frac{\text { usitatissimum }}{\text { fla }}$ & & Uncommon & Slope \\
\hline
\end{tabular}

OXALIDACEAE Oxalis family

Oxalis corniculata L. Creeping oxalis Woodsorrel

Uncommon

Gardens and shaded slope

GERANIACEAE Geranium family

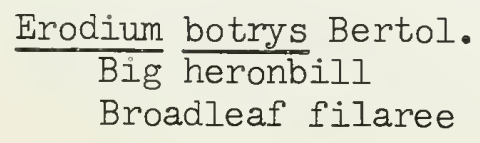

Broadleaf filaree

Abundant
Slope and dry swale


FORBS

\begin{tabular}{llll}
\hline Botanical and common name & $\begin{array}{l}: \\
:\end{array}$ & Occurrence & $\vdots$ \\
& $:$ & Habitat \\
\hline
\end{tabular}

Erodium cicutarium L'Her.

Alfileria

Common

Lower slope

Redstem filaree

Erodium obtusiplicatum (Maire,

Weiller and Wilczek) J.T. Howell

Very common Slope

(E. botrys f. montanum Brumhard)

Big heronbill

Broadleaf filaree

Erodium moschatum L'Her.

Musk heronbill

Uncommon

Lower slope

Whitestem filaree

Geranium carolinianum L.

Carolina geranium

Unc ommon

Lower slope

Geranium sphaerospermum Fern.

Geranium

Uncommon

Lower slope

LIMNANTHACEAE Meadowfoam family

Limnanthes douglasi $\mathrm{R} . \mathrm{Br}$. Douglas meadowfoam

Common

Creekbank

Limnanthes montana

Unc ommon

Creekbank

Mountain meadowfoam

EUPHORBIACEAE Spurge family

Euphorbia ocellata D. and $\mathrm{H}$. Euphorbia

Common

Slope

\section{ZYGOPHYLIACEAE Caltrops family}

Tribulus terrestris L.

Rare

Disturbed soil

Puncturevine

Goathead 


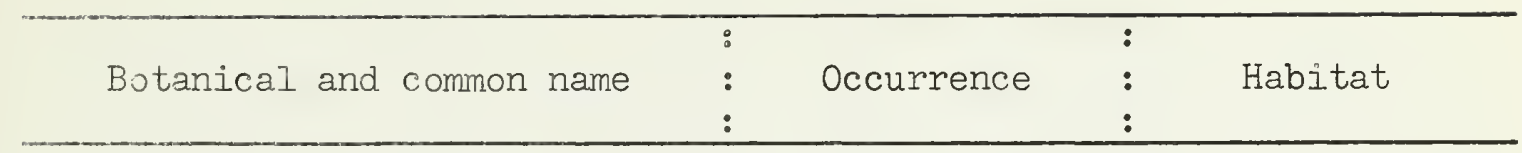

MALVACEAE Mallow family

Malva parviflora I.

Uncommon Disturbed soil

Little mallow

Cheeseweed

Sidalcea hartwegii Gray

Common

Lower slope and

Checkermallow swale

LOASACEAE Loasa family

Mentrelia lindleyi $\mathrm{T}$. and $\mathrm{G}$.

Rare

Slope, especially Lindley mentzelia south

DATISCACEAE Datisca family

Datisca glomerata Brew. and Wats。 Rare Creekbed Durangoroct

CUCURBITACEAE Gourd family

Cucurbita foetidissima H.B.K. Calabazillia

Rare

Slope

Echinocystis fabacea Naud. Mockcucumber

Common

Slope, climbing on shrubs

Echinocystis macrocarpa Greene Chilicothe mockcucumber

Common

Slope, climbing on shrubs

\section{IYTHRACEAE Loosestrife family}

Iythrum hyssipifolia L。 Hyssop lythrum

Common

Loosestrife

ONAGRACEAE Evening primrose family

Boisduvalia densiflora (Lindl。) Uncommon

Swale

Dense spikeprimrose 
FORBS

Botanical and common name $\begin{aligned}: & \text { Occurrence }: \\ & :\end{aligned}$

Boisduvalia stricta (Gray) Uncommon Swale

Greene

Brook spikeprimrose

Epilobium paniculatum Nutt.

Autumn willowweed

Clarkia dudleyana Abrams

(Godetia dudleyana Abrams)

Dudley's godetia

Clarkia elegans Dougl.

Rose clarkia

Uncommon

Slope

Common

Slope, especially north

Common

Slope, especially north

Clarkia nitens Lewis and Lewis (Godetia nitens)

Clarkia

Clarkia purpurea quadrivulnera

(DougI.) Lewis and Lewis

(Godetia quadrivuInera [Dougl.]

spach.)

Clarkia

Clarkia williamsonii (Durand and Hilgard) Lewis and Lewis

(Godetia viminea Spach.)

Orchid godetia

Oenothera dentata Cav.

Field primrose

Common

Slope

Uncommon

Slope

Common

Slope, especially north

Common

Slope, especially south

Oenothera graciflora Hook. and Arn.

Slender primrose

Common

Lower slope

UMBELLIFERAE Parsley family

Daucus pusillus Michx.

Southwestern carrot

Common

Slope

Rattlesnake weed

Eryngium globosum Jepson

Button snakeweed

Common

Swale 
FORBS

Botanical and common name :

Botanical and common name :

Lomatium utriculatum (Nutt.)

Colt and $\overline{\text { Rose }}$

(Cogswellia)

Common lomatium

Perideridia pringlei (C. and R.) Rare

Nels, and Macbr.

(Eulophus pringlei C. and R.)

Pringle's yampan

Sanicula bipinnatifida Dougl. Purple sanicle

Sanicula tuberosa Torr. Tuber sanicle

PRIMULACEAE Primrose family

Dodecatheon patulum Greene Dwarf shootingstar

GENTIANACEAE Gentian family

Centaurium venustum (Gray) Rob. Common Pink centaurium

\section{ASCLEPIADACEAE Milkweed family}

Asclepias cordifolia (Benth. Jepson

Purple milkweed

Asclepias mexicana Cav. Mexican milkweed

Asclepias vestita Hook。 and Arn. Common Wooly milkweed
Uncommon

Uncommon

Common

Slope

Common

Rock outcrop

Uncommon

Shaded slope

Dry swale

Uncommon Slope

Dry swale or lower slope

Slope 
FORBS

Botanical and common name

Occurrence $\begin{array}{lll} & \vdots \\ & :\end{array}$ Habitat

\section{POLEMONIACEAE Phlox family}

Eriastrum pluriflorum (Heller)

H. L. Mason

Uncommon

Slope, on

(Gilia brauntonii Jepson and shallow soil Mason)

Gilia

Gilia capitata Dougl. Globe gilia

Uncommon

Slope

Gilia tricolor Benth. Birdseye gilia

Very common

Slope

Linanthus bicolor (Nutt.) Greene Bicolored linanthus

Common

Slope

Linanthus ciliatus (Benth.)

Common

Slope

Bristlyaleaved linanthus

Linanthus dichotomus Benth. Eveningsnow linanthus

Uncommon

Slope, especially north

Linanthus filipes (Benth。)

Common

Slope, on shallow soil

Filiform linanthus

Linanthus serrulatus Greene Madera linanthus

Uncommon

Navarretia pubescens (Benth.)

Common

Hook. and Arn.

Downy navarretia

Slope, especially north

Slope, on shallow soil

HYDROPHYLLACEAE Phacelia family

Nemophila aurita Lindl. Purple nemophila

Uncommon

Shaded slope

Nemophila heterophylla F. and M. Common Shaded slope Small white nemophila 


\section{FORBS}

\section{Botanical and common name}

: Occurrence

Nemophila maculata Benth.

Spotted nemophila

Uncommon

Creekbank

Fivespot nemophila

Nemophila menziesii Hook. and

Arn.

Babyblue-eyes nemophila

Phacelia californica Cham.

California phacelia

Uncommon

Rock outcrop

Phacelia hispida Gray

Caterpillar phacelia

Common

Rock outcrop

Phacelia platyloba Gray

Uncommon

Lower slope

Broadlobed phacelia

BORAGINACEAE Borage family

Allocarya trachycarpa (Gray)

Greene

Common

Wet swale

Rough-fruited allocarya

Amsinckia douglasiana DC. Douglas fiddleneck

Common

Shaded slope

Amsinckia intermedia Fisch. and Mey.

Common

Slope

Fireweed fiddleneck

Amsinckia retrorsa Suksd.

Rigid fiddleneck

Uncommon Slope

Harvest fireweed

Cryptantha flaccida (Dougl.)

Greene

Common

Slope

Cryptantha

Cryptantha hooveri I. M. Johnston Uncommon

Slope

Hoover's cryptantha

Heliotropium curassauicum L.

Salt heliotrope

Rare

Disturbed soil 
FORBS

\begin{tabular}{lll}
\hline Botanical and common name & $:$ & Occurrence \\
\hline
\end{tabular}

Plagiobothrys canescens Benth. Valley popcornflower

Plagiobothrys nothofulvus Gray Redstain popcornflower

Plagiobothrys tenellus Gray Slender popcornflower

VERBENACEAE Verbena family

Verbena lasiostachys Link. (Verbena prostrata $\mathrm{R}, \mathrm{Br}$.) Western verbena

IABIATAE Mint family

Marrubium vulgare L。 Common hoarhound

Monardella candicans Benth. Sierra monardella

Monardella lanceolata Gray Mustang mint

Pogogyne douglasi Benth. Douglas pogogyne

Salvia carduacea Benth. Thistle sage

Salvia columbariae Benth. California chia

Scutellaria bolanderi Gray Bolander's skullcap

Scutellaria tuberosa Benth. Tuberous skullcap
Common

Very common

Common

Uncommon

Slope

Lower slope

and dry swale

Slope and dry swale

Slope, on shallow soil

Habitat

Uncommon

Disturbed soil

Common

Swale

Uncommon

Swale

Common

Swale

Common

Slope

Uncommon

Shaded slope

Uncommon

Wet swale and seeps

Uncommon

Shaded slope 


\section{FORBS}

\begin{tabular}{llll}
\hline \multirow{2}{*}{ Botanical and common name } & $\vdots$ & Occurrence & $\vdots$ \\
& $:$ & & Habitat \\
\hline
\end{tabular}

Stachys albens Gray Whit tehedge nettle

Trichostema lanceolatum Benth. Vinegar bluecurls

Trichostoma oblongum Benth. Mountain bluecurls

\section{SOLANACEAE Nightshade family}

Datura meteloides DC. Sacred datura

Tolguacha

Nicotiana attenuata Torr. Coyote tobacco

Solanum nigrum L. Black nightshade

Solanum xanthi Gray Purple nightshade

SCROPHULARIACEAE Figwort family

Antirrhinum cornutum Benth. Spurred snapdragon

Collinsia bartsiaefolia Benth. Seaside collinsia

Collinsia bicolor Benth. Pagoda collinsia Chinese houses

Collinsia tinctoria Hartw. Browns tain collinsia Sticky Chinese houses
Uncommon

Seeps

Common

Slope

Uncommon

Swale

Uncommon

Disturbed soil

Uncommon

Rare

Uncommon

Lower slope

Uncommon

Uncommon

Slope

Common

Shaded slope

Uncommon

Shaded creekbed 
FORBS

Botanical and common name

Occurrence $\begin{array}{ll}: & \\ & \vdots \\ \end{array}$

Cordylanthus compactus Pennell Muleweed

Uncommon

Rocky slope

Mimulus pilosus (Benth.) Wats. Downy mimulus

Uncommon Lower slope

Mimulus bolanderi Gray Tobacco mimulus

Uncommon

Shaded slope

Mimulus floribundus Dougl.

Uncommon

Wet swale Monkeyflower

Mimulus nasutus Greene

(Mimulus guttatus nasutus Jepson)

Common

Wet swale Common monkey flower

Mimulus nanus Hook. and Arn. Dwarf monkeyflower

Uncommon

Slope

Mimulus viscidus Congdon.

Uncommon

Slope Viscid monkeyflower

Mimulus pictus (Curran) Gray Painted monkeyflower

Uncommon

Slope

Orthocarpus erianthus micranthus

(Gray) Jepson

Common

Slope

Johnnytuck owlclover

Orthocarpus linearilobus Benth. Pallid owlclover

Common

Slope

Orthocarpus purpurascens Benth. Escobita owlclover

Common

Slope

Common owlclover

Penstemon breviflorus Lindl.

Stubflower penstemon

Common

Rocky slope

Scrophularia californica Cham. and Sch.

Uncommon

Rock outcrop

California figwort 
FORBS

\begin{tabular}{llll}
\hline Botanical and common name & $\vdots$ & $\vdots$ \\
& $\vdots$ & Occurrence & Habitat \\
\hline
\end{tabular}

Verbascum blattaria L. Moth mullein

Rare

Lower slope

PLANTAGINACEAE Plantain family

Plantago erecta Morris Dotseed plantain

Very common Slope

RUBIACEAE Madder family

Galium aparine L.

Catchweed bedstraw

Common

Shaded slope

or swale

Galium nuttalli Gray

Uncommon

Shaded slope

Nuttall bedstraw

or swale

\section{VAIERIANACEAE Valerian family}

Plectritis ciliosa (Greene)

Common

Plectritis

Plectritis macrocera $T$. and $G$. Longhorn plectritis

Uncommon

Shady lower slope

or creekbank

Shady lower

slope

CAMPANULACEAE Bellflower family

Githopsis specularioides Nutt. Githopsis

Uncommon Slope

LOBELIACEAE Lobelia family

Downingia bicornuta Gray Downingia

Uncommon

Wet swale

Nemacladus rigidus interior

Munz

Nemacladus

Uncommon Lower slope

or swale 


Botanical and common name : Occurrence : Habitat

COMPOSITAE Sunflower family

Achillea lanulosa I.

Uncommon

Lower slope Western yarrow

Agoseris heterophylla (Nutt.)

Common

Slope

Greene

Annual agoseris

Anaphalis margaritacea (L.)

Uncommon

Rocky slope

$\overline{\mathrm{B}}$. and $\mathrm{H}_{\text {. }}$

Common pearleverlasting

Baeria chrysostoma gracilis Hall Branchy goldfields

Baeria uliginosa (Nutt.) Gray Goldfields

Balsamorhiza deltoidea Nutt. Balsamroot

Bidens frondosa $L$.

Devils beggarticks

Very common

Slope

Uncommon

Slope

Rare

North slope

Brickellia california T. and G. Californica brikellia

Calycadenia mollis Gray Rosinweed

Calycadenia multiglandulosa DC. Rosinweed

Uncommon

Lower slope

Uncommon

Rocky slope

Common

Slope

Common

Slope

$\frac{\text { Calycadenia }}{\text { Rosinweed }} \frac{\text { villosa }}{D C}$.

Uncommon

Slope

Centaurea melitensis L.

Common

Slope

Malta centaurea

Tocalote

Napa thistle

Centromadia pungens ( $T$. and G.) Uncommon

Lower slope

Greene

Common spikeweed 


\section{FORBS}

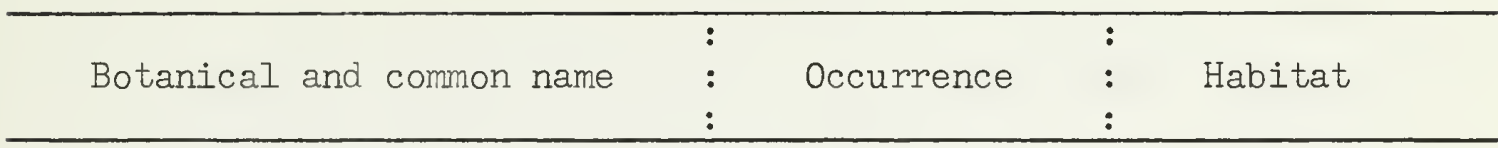

Chaenactis glabriuscula DC. Chaenactis

Common

Slope

Cirsium occidentale (Nutt.)

Jepson

Common

Slope

Western thistle

Coreopsis bigelovi (Gray) Hall Bigelow coreopsis

Unc ommon

Shaded slope

Coreopsis douglasi (DC.) Hall Douglas coreopsis

Uncommon

Shaded slope

Coreopsis stillmani (Gray)

Uncommon

Shaded slope

Stillman coreopsis

Erigeron canadensis L.

Uncommon

Disturbed soil

Horseweed fleabane

Erigeron foliosus stenophyllus

(Nutt.) Gray

Uncommon

Rocky slope

Leafy fleabane

Eriophyllum confertiflorum Gray Goldenyarrow eriophyllum

Common

Rocky slope

Evax caulescens Benth.

Uncommon

Slope, on shallow soil

Filago gallica L. Fluffweed

Common

Slope

Gnaphalium californicum Gray California everlasting

Gnaphalium palustre Nutt. Lowland cudweed

Uncommon

Rocky slope

Uncommon

Lower, slope

Grindelia camporum Greene

Uncommon

Slope, especially south 
FORBS

Botanical and common name $\begin{aligned} & : \\ & :\end{aligned}$

: Habitat

:

Hemizonia heermannii

(Hemizonia virgata heermannii

Very common

Lower slope

and swale

Jepson)

Yellow tarweed

Hemizonia kelloggii Greene

(Hemizonia wrightii Gray)

Wright's tarweed

Hypochoeris glabra $I$.

Very common

Smooth catsear

Common

Rocky slope

Lactuca serriola

(Iactuca Scariola L.)

Uncommon

Lower slope and dry swale

Disturbed soil

Prickly lettuce

Layia gaillardioides Hook.

and Arn.

Layia

Layía pentachaeta hansenii

(Jepson) Keck

Layia

Tidytips

Lessingia germanorum vallicola

Common

Slope

Lessingia

Lessingia sp. Lessingia

Common

Shady slope

Common

Shady slope

Madia elegans densifolia Jepson Madia

Matricaria matricarioides (Less.) Uncommon

Common

Slope

Porter

Pineappleweed

Micropus californicus F. and M. Common Micropus

Uncommon

Creekbed

Disturbed soil

Pentachaeta exilis Gray

Common

Slope, on shallow soil

Pentachaeta

Slope, on

shallow soil 


\section{FORBS}

\begin{tabular}{|c|c|c|}
\hline Botanical and common name & Occurrence & Habitat \\
\hline$\frac{\text { Senecio douglasi }}{\text { Douglas groundsel }}$ & Uncommon & Slope \\
\hline$\frac{\text { Silybum marianum }}{\text { Milkthistle }}\left(L_{\bullet}\right)$ Gaertn. & Rare & Creekbank \\
\hline $\begin{array}{l}\text { Stephanomeria pauciflora (Torr.) } \\
\begin{array}{l}\text { Nels. } \\
\text { Wirelettuce }\end{array}\end{array}$ & Uncommon & Slope \\
\hline$\frac{\text { Uropappus }}{\text { Uropappus }}$ & Uncommon & Lower slope \\
\hline$\frac{\text { Xanthium }}{\text { Cockl }} \frac{\text { canadense }}{\text { ebur }}$ Mill. & Uncommon & Creekbed \\
\hline
\end{tabular}


WOODY PLANTS

\begin{tabular}{llll}
\hline Botanical and common name & $:$ & $:$ & \\
& $:$ & Occurrence & Habitat \\
\hline
\end{tabular}

\section{PINACEAE Pine family}

Pinus sabiniana Dougl. Digger pine

SALICACEAE Willow family

Populus fremontii Wats. Fremont cottonwood

Salix laevigata Bebb. Red willow

FAGACEAE Oak family

Quercus douglasii Hook, and Arn. Blue oak

Quercus morehus Kell. Oracle oak

Quercus wislizenii DC. Interior live oak

LORANTHACEAE Mistletoe family

Arceuthobium campylopodum Engelm. Western dwarf mistletoe Pine mistletoe

Phoradendron villosum Nutt. Common mistletoe

ANACARDIACEAE Sumac family

Rhus diversiloba $T$. and G. Pacific poisonoak
Very common Slope

Uncommon

Creekbank

Uncommon

Creekbank

Rare

Slope

Very common Slope

Common

Parasitic on digger pine

Very common

Parasitic on blue oak and live oak

Common

Rocky slope 
WOODY PIANTS

\begin{tabular}{llll}
\hline Botanical and common name & $\vdots$ & Occurrence & $\vdots$ \\
& $:$ & Habitat \\
\hline
\end{tabular}

Rhus trilobata Nutt。 Skunkbush sumac

Rare

Slope

Squawbush

SAPINDACEAE Buckeye family

Aesculus californica (Spach) Nutt. Common Slope California buckeye

RHAMNACEAE Buckthorn family

Ceanothus cuneatus (Hook.) Nutt. Very common Buckbrush ceanothus Wedgeleaf ceanothus

Ceanothus leucodermis Greene

(Ceanothus divaricatus)

Chaparral whitethorn ceanothus

Rhamnus californica cuspidata

(Greene) Wolf

California buckthorn

California coffeeberry

Rhamnus crocea ilicifolia Greene Common Slope Hollyleaf buckthorn

Redberry buckthorn

ERICACEAE Heath family

Arctostaphlos mariposa Dudley Common Rocky slope

HYDROPHYLLACEAE Phacelia family

Eriodictyon californicum

Uncommon

Slope, especially north

Uncommon Slope

Common

Slope

(Hook. and Arn.) Greene

Yerba santa 
WOODY PLANTS

\begin{tabular}{llll}
\hline Botanical and common name & $:$ & $\mathbf{0}$ & $\mathbf{0}$ \\
& $\mathbf{0}$ & Occurrence & \\
\hline
\end{tabular}

RUBIACEAE Madder family

Cephalanthus occidentalis L.

Uncommon Seep

Common buttonbush

CAPRIFOLIACEAE Honeysuckle family

Lonicera interrupta Benth.

Chaparral honeysuckle

Räre

Slope, especially north

Sambucus caerulea Raf.

Uncommon

Rocky slope

(Sambucus glauca Nutt.)

Blueberry elder

Blue elderberry 

